\title{
Riesgo de depresión, alcoholismo, tabaquismo y consumo de sustancias psicoactivas en personal de enfermería, de dos instituciones hospitalarias del área metropolitana de la ciudad de Medellín
}

Fecha de recibido: 14 de noviembre de 2016 Fecha de aprobación: 20 de diciembre de 2016

Forma de citar este artículo: Londoño J, Chica OP, Marín IC. Riesgo de depresión, alcoholismo, tabaquismo y consumo de sustancias psicoactivas en personal de enfermería, de dos instituciones hospitalarias del área metropolitana de la ciudad de Medellín. Med U.P.B. 2017;36(1):34-43. DOI:10.18566/medupb.v36n1.a05

1. Universidad de Antioquia, Facultad de Enfermería. Enfermera, Magíster en Epidemiología. Docente, Grupo Salud de las mujeres. Medellín, Colombia.

2. Universidad de Antioquia, Facultad de Enfermería. Enfermera, Especialista en Gestión de la Calidad y Auditoría en Salud. Docente, Grupo Salud de las mujeres. Medellín, Colombia.

3. Universidad de Antioquia, Facultad de Enfermería. Estudiante de Enfermería VIII semestre, Grupo salud de las mujeres. Medellín, Colombia.
Risk of depression, alcoholism, smoking, and psychoactive substance use among nursing personnel at two institutions in the metropolitan area of Medellin / Risco de depressão, alcoolismo, tabaquismo e consumo de substâncias psicoativas no pessoal de enfermagem, de duas instituições hospitalares da área metropolitana da cidade de Medellín

Johanna Londoño Restrepo ${ }^{1}$, Olga Patricia Chica Álvarez², Isabel Cristina Marín Agudelo³

\section{RESUMEN}

Objetivo: describir el riesgo de depresión, alcoholismo, tabaquismo, consumo de sustancias psicoactivas y variables asociadas con el consumo en personal de enfermería de dos instituciones hospitalarias del área metropolitana de Medellín.

Metodología: estudio transversal, en el que se exploran características sociodemográficas y de consumo. Se aplicó la escala de Zung para determinar riesgo de depresión, la escala CAGE para riesgo de alcoholismo y el test de Fagerstrom para adicción al cigarrillo. Para identificar la prevalencia de consumo en vida de sustancias psicoactivas se usó el autoreporte. Se realizó análisis bivariado para explorar variables asociadas con el consumo. La muestra se constituyó por 157 enfermeras y auxiliares de enfermería de los servicios de cirugía y urgencias. Los datos se analizaron en el programa estadístico SPSS, versión 20. Resultados: el 9.5\% del personal de enfermería tiene riesgo de depresión. El $67.5 \%$ ha consumido alguna vez en su vida una sustancia psicoactiva y el alcohol fue la sustancia más consumida con el $65 \%$, seguido por: marihuana, alucinógenos, sedantes y benzodiacepinas sin indicación médica. Se halló que en el caso de los hombres se aumenta el riesgo de consumir sustancias psicoactivas (OR 11.07).

Conclusión: el consumo de sustancias psicoactivas, el riesgo de alcoholismo, el tabaquismo y la depresión fueron menores y similares a otros estudios específicos en personal de salud, mientras que el estrés y la despersonalización presentaron mayor prevalencia. Palabras clave: salud mental; enfermería; trastornos relacionados con el uso de sustancias psicoactivas; depresión; recursos humanos.

\section{ABSTRACT}

Objective: To describe the risk of depression, alcoholism, smoking, and psychoactive substance use and the variables associated to abuse in nursing personnel at two hospitals in the metropolitan area of Medellin. 
Methodology: Cross-sectional study that explores sociodemographic and abuse characteristics. The Zung scale was administered to determine risk of depression, CAGE questionnaire to evaluate risk of alcoholism, and Fagerstrom test to gauge smoking addiction. Self-report was used to identify prevalence of substance abuse. Bivariate analysis was conducted to explore variables associated to abuse. The sample included 157 operating room and emergency room nurses and auxiliary nurses. Data were analyzed using SPSS, version 20 software.

Results: Of all nursing personnel, 9.5\% had risk of depression while $67.5 \%$ had used some sort of psychoactive substance. Alcohol was the most common (65\%), followed by marihuana, hallucinogens, sedatives, and benzodiazepines without medical prescription. It was found that males presented a higher risk of psychoactive substance abuse (OR 11.07). Conclusion: Risk of psychoactive substance abuse, alcoholism, smoking, and depression were lower and similar to other studies conducted in healthcare personnel, whereas stress and depersonalization had a higher prevalence.

Keywords: mental health; nursing; substance-related disorders; depression; manpower.

\section{RESUMO}

Objetivo: descrever o risco de depressão, alcoolismo, tabaquismo, consumo de substâncias psicoativas e variáveis associadas com o consumo no pessoal de enfermagem de duas instituições hospitalares da área metropolitana de Medellín.

Metodologia: estudo transversal, no qual se exploram características sócio-demográficas e de consumo. Se aplicou a escala de Zung para determinar o risco de depressão, a escala CAGE para risco de alcoolismo e o teste de Fagerstrom para adição ao cigarro. Para identificar a prevalência de consumo em vida de substâncias psicoativas se usou o auto-relatório. Se realizou análise bivariado para explorar variáveis associadas com o consumo. A amostra se constituiu por 157 enfermeiras e auxiliares de enfermagem dos serviços de cirurgia e urgências. Os dados se analisaram no programa estatístico SPSS, versão 20.

Resultados: $9.5 \%$ do pessoal de enfermagem tem risco de depressão. $67.5 \%$ há consumido alguma vez na sua vida uma substância psicoativa e o álcool foi a substância mais consumida com $65 \%$, seguido por: maconha, alucinógenos, sedantes e benzodiazepina sem indicação médica. Se encontrou que no caso dos homens se aumenta o risco de consumir substâncias psicoativas (OR 11.07).

Conclusão: o consumo de substâncias psicoativas, o risco de alcoolismo, o tabaquismo e a depressão foram menores e similares a outros estudos específicos na pessoal de saúde, enquanto que o estresse e a despersonalização apresentaram maior prevalência. Palavras chave: saúde mental; enfermagem; transtornos relacionados com o uso de substâncias psicoativas; depressão; recursos humanos.

\section{INTRODUCCIÓN}

Los trastornos mentales aportan, de forma significativa, en la carga de la enfermedad. De acuerdo con las estadísticas de salud mundial y el monitoreo de salud para los objetivos de desarrollo sostenible (2016), la depresión y la ansiedad son los trastornos más prevalentes. Se estima que una de cada 10 personas en el mundo las padecen y tienen, por tanto, un importante riesgo suicida (segunda causa de muerte en el grupo de personas de 15 a 29 años de edad $)^{1}$. Por otra parte, los trastornos por uso de sustancias y el consumo de sustancias representan un problema para la salud pública, en particular, los que tienen qué ver con el alcohol por su relación con enfermedades cardiovasculares, diabetes, enfermedades psiquiátricas, oncológicas y accidentes de tránsito. En 2010 el 38\% de la población de 15 años o más había bebido alcohol durante el último año ${ }^{2}$. Por otra parte, el consumo de tabaco es uno de los
Dirección de

correspondencia: Johanna Londoño Restrepo. Correo electrónico: johana.

londono@udea.edu.co 
principales factores de riesgo para enfermedades crónicas, aunque su consumo ha disminuido pero las estadísticas se mantienen estables en el hemisferio asiático ${ }^{3}$.

Según la Encuesta Nacional de Salud Mental (2015), en Colombia el 9.6\% de las personas tienen psicopatología probable sin un diagnóstico comprobado, y es mayor en mujeres (10.8\%). Al indagar por sustancias psicoactivas, las más consumidas son: cannabis, opioides, cocaína, anfetaminas y las lícitas como el alcohol, con una prevalencia en vida de $57.6 \%$ y un consumo diario de cigarrillo del $47.8 \%$. Los factores de riesgo asociados con el consumo son la edad, el ser hombre, el desempleo, el consumo entre amigos y el fácil acceso ${ }^{4}$.

En Medellín, el Primer estudio poblacional de salud mental, reportó una prevalencia en vida de uno o más trastornos mentales del $26.6 \%$, en relación con el comportamiento de los trastornos en los últimos 12 meses. La depresión se presentó en un 4.1\%; trastorno por uso de sustancias psicoactivas 5.2\%; abuso a alcohol 3.6\%; abuso de drogas $1.8 \%$ e intento suicida $4.6 \% 5$.

Los trastornos mentales impactan las diferentes esferas sociopolíticas y familiares, las secuelas económicas son evidentes con el paso de los años porque padecer un trastorno de estos conlleva ausentismo laboral y, por ende, disminución en la productividad y en el logro de metas personales, familiares y en el desempeño de roles sociales, educativos y laborales. De acuerdo con los estudios, las personas con depresión pierden un $20 \%$ de su tiempo en el trabajo y están expuestos a sufrir accidentes laborales ${ }^{4}$. Entre las profesiones y ocupaciones con riesgo de sufrir trastornos mentales y consumo de sustancias psicoactivas, se encuentran las de régimen irregular de horario, intensa vida social y con desarraigo familiar ${ }^{6}$.

En Colombia, según el reporte del consumo de sustancias psicoactivas en la población económicamente activa, el $20.8 \%$ de los hombres presenta trastorno por uso de sustancias alguna vez en la vida, mientras que las mujeres tienen una frecuencia del $2.6 \%{ }^{7}$. Otros estudios que aluden al consumo de sustancias psicoactivas en trabajadores de la salud describen que el personal médico está entre los profesionales con mayor consumo de opioides y benzodiacepinas 6 .

Respecto a las prevalencias-vida del personal de enfermería, en Brasil reportan que el $54.3 \%$ presenta trastorno del ánimo; $28.7 \%$ trastornos relacionados con el estrés y somáticos; $5.5 \%$ trastornos mentales y de comportamiento por uso de sustancias psicoactivas. Del personal de enfermería encuestado, el $49.6 \%$ pertenecía al servicio de urgencias; $22.8 \%$ a hospitalización; $16 \%$ a servicios de psiquiatría y $4.8 \%$ al servicio de ginecobstetricia. Los enfermeros expresan que el fácil acceso a psicofármacos los hace más susceptibles de padecer abuso y adicción. Según grupos etarios los trabajadores entre 30 y 49 años eran los que más consultaban por estas patologías y las auxiliares de enfermería son las más afectadas por dependencia y abstinencia a alcohol, opioides y cigarrillo ${ }^{8,9}$.

En Medellín, el estudio de prácticas saludables en médicos y personal de enfermería de una institución hospitalaria identificó que el $56 \%$ de los médicos y el $69.4 \%$ de los enfermeros consumieron licor en forma periódica, en especial, durante las reuniones sociales con sus colegas ${ }^{10}$.

Se realizó un estudio trasversal con el objetivo de estimar la prevalencia de consumo de sustancias psicoactivas, riesgo de alcoholismo, tabaquismo, autoreporte de estrés y riesgo de depresión de acuerdo con la escala de Zung; así como explorar la asociación de variables sociodemográficas con el consumo de sustancias psicoactivas en el personal de enfermería de los servicios de cirugía y urgencias de dos instituciones hospitalarias del área metropolitana de Medellín.

\section{METODOLOGÍA}

\section{Tipo de estudio}

Estudio transversal para determinar la prevalencia de consumo de sustancias psicoactivas, riesgo de alcoholismo, tabaquismo, autoreporte de estrés y riesgo de depresión de acuerdo con la escala de Zung y la asociación de variables sociales y mentales con el consumo de sustancias psicoactivas entendidas como sustancias lícitas e ilícitas, en 47 enfermeras y 110 auxiliares de enfermería en los servicios de cirugía y urgencias de dos instituciones hospitalarias del área metropolitana de Medellín.

\section{Lugar y período de estudio}

El estudio se realizó en dos instituciones hospitalarias de tercer nivel de atención del Valle de Aburrá (ciudad de Medellín) y la recolección de la información se llevó a cabo durante el año 2015.

\section{Selección y muestra}

La población de referencia fue el personal de enfermería de ambas instituciones, se incluyeron enfermeros que laboraban en los servicios de urgencias y cirugía con contratación mayor a tres meses, que culminaron el período de prueba y se excluyó personal que fue trasladado recientemente al servicio, personas pertenecientes a otros servicios y quienes participaron en la prueba piloto. Se hace muestreo estratificado por los servicios de cirugía y urgencias y el cálculo de la muestra se hizo según la fórmula establecida para estudios descriptivos: 
$\mathrm{n}=\mathrm{NXZXPXQ} / \mathrm{d} 2 \mathrm{x}(\mathrm{N}-1)+\mathrm{Z} 2 \mathrm{XPXQ}=164$ trabajadores de salud (enfermeras, auxiliares de enfermería) donde: $\mathrm{N}=$ Total de la población: 285 (enfermeras, auxiliares de enfermería de los servicios de cirugía y urgencias), $Z$ $=1.96$ al cuadrado (confianza del 95\%), $\mathrm{p}=$ proporción esperada (50\% se utiliza debido a que se desconoce la prevalencia de consumo y depresión en el personal de enfermería en Medellín), $\mathrm{q}=($ en este caso 1-0.50 = 0.50), $\mathrm{d}=$ precisión $(5 \%)$.

El muestreo se hizo estratificado para garantizar una asignación proporcional de tamaño muestral a los diferentes estratos correspondientes a las diferentes ocupaciones de los trabajadores de la salud, enfermeras y auxiliares de enfermería. Ksh $=\mathrm{n} / \mathrm{N}=164 / 285=$ 0.58 . El total de la subpoblación se multiplicó por la fracción constante para obtener el tamaño de la muestra por estrato $(\mathrm{Nh})(\mathrm{fh})=\mathrm{nh}$. De las 164 encuestas recolectadas, se anularon siete, tres de ellas por no cumplir con los criterios de inclusión y cuatro por información insuficiente.

\section{Instrumento y procedimiento de recolección de la información}

Las investigadoras aplicaron un cuestionario que estaba compuesto por variables sociodemográficas, la escala de Zung abreviada y validada para Colombia con un alfa de Cronbach de 0.845 , sensibilidad 0.92, especificidad 0.71 , un área bajo de la curva ROC de 0.84 y un punto de corte de 22. Es un instrumento autoadministrado que valora el riesgo de depresión, consta de 10 preguntas, en escala Likert de 0 a 4 y un puntaje superior a 22 sugiere que la persona tiene riesgo de presentar un trastorno depresivo. El instrumento fue validado por Díaz et al ${ }^{11}$.

Se indagó por consumo de sustancia psicoactiva a través de autoreporte de una variable categórica (sí/no) y la prevalencia en vida, anual, mensual y semanal de consumo discriminado por sustancia. No se aplicó un instrumento para detectar riesgo de dependencia a sustancias diferentes al tabaco y el alcohol. Se aplicó, para la detección de adicción al alcohol, el test CAGE que consta de cuatro ítems y se explora por tres aspectos subjetivos de la persona en relación con el consumo del alcohol y el último ítem, aspectos relacionados con la abstinencia alcohólica. Puntajes de cuatro indican dependencia al alcohol, uno a tres en riesgo y cero no dependiente. Este test tiene 0.65 de sensibilidad y 0.80 de especificidad. Por otra parte, para la adicción a cigarrillo se utiliza el test de Fagerstrom, que tiene una especificidad del 0.88 y una sensibilidad del $94 \%$, consta de seis preguntas, cuatro de ellas se responden con sí o no y las demás con escala de Likert de 0 a $4^{12}$.

\section{Plan de análisis}

Se hizo un análisis univariado de las características sociodemográficas y mentales de los trabajadores; para las variables cualitativas los datos se expresaron en medidas de frecuencia relativas y absolutas; las variables cuantitativas por medidas de tendencia central y dispersión, se expresó la mediana con su respectivo rango intercuartil por cuanto la variable edad no distribuyó normal. Se ejecutó un análisis bivariado para encontrar asociación entre el consumo de sustancias psicoactivas con las variables sexo, edad, servicio de trabajo, profesión, riesgo de depresión, autoreporte de estrés, antecedente de trastorno mental y estado civil; para evaluar la fuerza de asociación entre las variables cualitativas se utilizó el odds ratio (OR), con su respectivo IC del 95\%.

La investigación fue de mínimo riesgo, según Resolución 008430 de 1993 y declaración de Helsinki. Se solicitó aval de la organización y del Comité de Ética, se explicó el propósito de la investigación al personal de enfermería y se firmó el consentimiento informado.

\section{RESULTADOS}

En la Tabla 1 se muestran las características sociodemográficas del personal de enfermería, se identificó que la persona más joven tenía 20 años, el mayor 58 años, la mediana para la edad fue de 32 años (RIC 12). El estado civil más frecuente fue soltero $62.4 \%$, seguido por casado en un $19.7 \%$. El 70.1\% está compuesto por auxiliares de enfermería; el 56.1\% del total del personal pertenecía a urgencias y el $43.9 \%$ a cirugía. El $16.6 \%$ realizaba un estudio de postgrado, pregrado o curso de educación no formal; el tipo de contratación del personal era a término indefinido; al preguntar por el desarrollo de actividades laborales fuera de la institución de salud el 4.5 \% afirmó realizarlo.

Al indagar por autoreporte de estrés el 55.4\% manifestó que su trabajo le genera estrés y es mayor esta frecuencia en el servicio de urgencias y en profesionales de enfermería; el $47.1 \%$ del personal declaró afectarle emocionalmente el estado de salud y la situación socioeconómica de los pacientes.

En la Tabla 2 se describen las acciones que ejecutan los enfermeros ante situaciones de estrés y ansiedad desencadenadas en las jornadas laborales. Para mejorar su estado de ánimo El personal de enfermería recurrió a: dialogar con sus compañeros sobre la situación vivida (82.8\%), dialogar con su familia (73.2\%), realizar actividades lúdicas $(60.5 \%)$, consumir psicofármacos para calmar la ansiedad (3.8\%), consumir sustancias psicoactivas (3.2\%) 
Tabla 1. Características generales de los participantes.

\begin{tabular}{lc}
\hline \multicolumn{1}{c}{ Característica } & n (\%) \\
\hline Edad & \\
$20-30$ años & $94(59.9)$ \\
$31-40$ años & $(22.9)$ \\
$41-50$ años & $18(11.5)$ \\
$>50$ años & $9(5.7)$ \\
Sexo & \\
Femenino & $122(77.7)$ \\
Estado civil & \\
Soltero & $98(62.4)$ \\
Casado & $31(19.7)$ \\
Unión libre & $26(16.6)$ \\
Viudo & $2(1.3)$ \\
Servicio & \\
Urgencias & $88(56.1)$ \\
Cirugía & $69(43.9)$ \\
Cursaban estudios & $26(16.6)$ \\
Pregrado & $17(10.8)$ \\
Postgrado & $5(3.2)$ \\
Cursos y/o diplomados & $3(1.9)$ \\
Técnica & $1(0.6)$ \\
Actividades que realiza en el tiempo libre & \\
Deportivas & $69(43.9)$ \\
Viajes & $23(14.6)$ \\
Estudios & $23(14.6)$ \\
Artísticas & $10(6.4)$ \\
Ninguna & $32(20.4)$ \\
\hline
\end{tabular}

y el (12.7\%) prefiere quedarse callado. Al comparar los servicios el $5.7 \%$ del personal de urgencias consumió sustancias psicoactivas y el $4.5 \%$ consumió psicofármacos para contrarrestar la ansiedad, a diferencia del servicio de cirugía en el que el consumo de psicofármacos fue del $2.9 \%$.

Según el autoreporte de antecedentes personales de enfermedad mental el 10\% fue diagnosticada en los últimos años con algún trastorno y de estos los prevalentes fueron: depresión (50\%), trastorno del sueño (25\%), ansiedad (19\%),y trastorno de déficit de atención e hiperactividad (TDAH) (6\%). Fue mayor el antecedente de trastorno mental en el personal de cirugía que en el de urgencias: el 5.8\% del personal de cirugía padeció depresión, 4.3\% trastorno del sueño y $2.9 \%$ ansiedad. Según la escala de Zung utilizada para diagnosticar riesgo de depresión (que indaga si durante los últimos 15 días hubo manifestaciones de tristeza, labilidad emocional, irritabilidad, disfrute de las actividades cotidianas, concentración laboral, entre otros), se encontró que el 9.5\% del personal de enfermería tiene riesgo de depresión y es mayor en los profesionales de enfermería respecto al personal auxiliar, OR 2.04 (IC: 0.787-5.322).

En la Tabla 3 se detalla la prevalencia de consumo de las drogas lícitas e ilícitas durante el último año, último mes, última semana y día anterior. El 67.5\% del personal ha consumido alguna vez en su vida una sustancia psicoactiva. El personal de urgencias consumió sedantes sin indicación médica $(2.3 \%)$, sedantes con indicación médica (3.4\%) e hipnóticos (1.1\%) a diferencia del servicio de cirugía en el que no se documentaron casos.

En cuanto al consumo de sustancias lícitas, la droga de mayor consumo fue el alcohol en un 65\% ( $\mathrm{n}=102)$; de los consumidores de alcohol 73 personas respondieron el test de CAGE y se encontró que el $93.2 \%$ es bebedor social

Tabla 2. Distribución de las acciones de respuesta del personal de enfermería ante situaciones de estrés por servicios de trabajo.

\begin{tabular}{lccccc}
\hline \multicolumn{1}{c}{ Variable } & & \multicolumn{2}{c}{ Cirugía } & \multicolumn{2}{c}{ Urgencias } \\
& & $\mathbf{n}$ & $\%$ & $\mathbf{n}$ & $\%$ \\
\hline Manifestación de sentimientos de estrés ante compañeros de trabajo & $\mathrm{Si}$ & 55 & 79.7 & 75 & 85.2 \\
& $\mathrm{No}$ & 14 & 20.3 & 13 & 14.8 \\
Consumo de SPA para contrarrestar el estrés & $\mathrm{Si}$ & 0 & 0 & 5 & 5.7 \\
No manifestación de sentimientos de estrés & $\mathrm{No}$ & 69 & 100 & 83 & 94.3 \\
& $\mathrm{Si}$ & 11 & 15.9 & 9 & 10.2 \\
Uso de psicofármacos para contrarrestar el estrés & No & 58 & 84.1 & 79 & 89.8 \\
& $\mathrm{Si}$ & 2 & 2.9 & 4 & 4.5 \\
& $\mathrm{No}$ & 67 & 97.1 & 84 & 95.5 \\
\hline
\end{tabular}


Tabla 3. Prevalencia de consumo de sustancias por año, mes, semana y día, según el tipo de droga consumida.

\begin{tabular}{|c|c|c|c|c|c|c|}
\hline Variable & $\begin{array}{c}\text { Más de } \\
\text { un año } \\
\text { n (\%) }\end{array}$ & $\begin{array}{l}\text { Último } \\
\text { año } \\
\text { n (\%) }\end{array}$ & $\begin{array}{c}\text { Último } \\
\text { mes } \\
\text { n (\%) }\end{array}$ & $\begin{array}{c}\text { Última } \\
\text { semana } \\
\text { n (\%) }\end{array}$ & $\begin{array}{l}\text { Último } \\
\text { día } \\
\text { n (\%) }\end{array}$ & $\begin{array}{l}\text { Total } \\
\text { n (\%) }\end{array}$ \\
\hline Alcohol & $5(4.9)$ & $14(13.7)$ & $45(44.1)$ & $33(32.4)$ & $5(4.9)$ & $102(65)$ \\
\hline Cigarrillo & $23(71)$ & $2(6.3)$ & $1(3.1)$ & $2(6.3)$ & $4(12.5)$ & $32(20.4)$ \\
\hline Bebidas energizantes & $5(11.1)$ & $8(17.8)$ & $9(20)$ & $16(35.6)$ & $7(15.6)$ & $45(28.7)$ \\
\hline Marihuana & $8(66.7)$ & $3(25)$ & $1(8.3)$ & - & - & $12(7.6)$ \\
\hline Alucinógenos/ hongos & $4(80)$ & $1(20)$ & - & - & - & $5(3.1)$ \\
\hline Sedantes: indicación médica & $2(66.7)$ & - & $1(33.3)$ & - & - & $3(1.9)$ \\
\hline Cocaína & $2(100)$ & - & - & - & - & $2(1.3)$ \\
\hline Sedantes: sin indicación médica & $1(50)$ & - & $1(50)$ & - & - & $2(1.3)$ \\
\hline Benzodiacepinas sin indicación médica & - & - & - & $1(100)$ & - & $1(0.6)$ \\
\hline Hipnóticos & $1(100)$ & - & - & - & - & $1(0.6)$ \\
\hline
\end{tabular}

(no dependientes) $5 \%$ tiene consumo de riesgo y $1.4 \%$ consumo perjudicial. El $8.3 \%$ del personal de cirugía tiene un consumo de riesgo y el $2.6 \%$ del personal de urgencias un consumo perjudicial.

En relación con el consumo de bebidas energizantes, el $28.7 \%$ lo ha consumido alguna vez en su vida. Por otra parte, el 20.4\% ( $\mathrm{n}=32)$ ha fumado cigarrillo alguna vez en su vida, $6.3 \%$ personas con consumo los últimos seis meses al responder el test de Fagerstrom, tienen una dependencia leve a la nicotina.

En la Tabla 4 se presenta la relación de algunas variables sociodemográficas y mentales con el consumo de sustancias psicoactivas lícitas e ilícitas en el personal de enfermería encuestado. Al evaluar los resultados del análisis bivariado, se encontró que el ser hombre implica un riesgo 10 veces mayor de consumir sustancias psicoactivas con respecto al de ser mujer. En relación con las variables edad, servicio de trabajo, profesión, riesgo de depresión, autoreporte de estrés y antecedente de trastorno mental no se encontraron diferencias estadísticamente significativas.

\section{DISCUSIÓN}

E1 trabajo de enfermería está inmerso en diversas complejidades que supone el cuidado de la salud del individuo y colectivo, el cual depende de la capacidad de cada uno de los trabajadores para actuar y responder ante situaciones de sufrimiento, dolor, enfermedad y muerte que atraviesa cada paciente; por otra parte, la exposición a factores ambientales laborales y familiares como turnos nocturnos, rotación del personal, demanda del sector salud, sobrecarga laboral, responsabilidades y preocupaciones excesivas, predisponen al equipo de enfermería a padecer trastornos mentales como depresión y consumir sustancias psicoactivas para controlar el estrés y ansiedad ${ }^{13}$.

En el estudio, al 55.4\% de los enfermeros el trabajo le genera estrés, lo que puede afectar directamente su estado emocional, percepción de la realidad, desencadenar fatiga $\mathrm{y}$ constituirse en un factor de riesgo para el consumo de sustancias psicoactivas, enfermedades mentales y crónicas como depresión, infarto de miocardio, hipertensión arterial, enfermedad ácido péptica y colon irritable.

Chiang et $\mathrm{al}^{14}$, menciona que las enfermeras que laboran en los servicios de urgencias y en unidades de cuidados intensivos tienen mayor riesgo de presentar episodios de estrés y dificultades para enfrentar los problemas, por la alta tensión a la que son sometidas, asegura que trabajar en servicios con mayor flujo y rotación de pacientes representa mayor nivel de estrés y de despersonalización atribuidos al intento del personal de compensar la carga laboral a la que están expuestos. En este estudio la prevalencia de despersonalización fue superior a la reportada por Freitas et al., con el $(47.6 \%)^{14,15}$. Se ha documentado que las personas que trabajan en los servicios de urgencias y cirugía tienen riesgo de consumir sustancias psicoactivas por la sobrecarga laboral a la que están expuestas; sin embargo, en el presente estudio no se halló una asociación significativa entre trabajar en estos servicios y consumir sustancias ${ }^{16}$. 
ARTÍCULO ORIGINAL - ORIGINAL ARTICLE - ARTIGO ORIGINAL | Riesgo de depresión, alcoholismo, tabaquismo y consumo de sustancias psicoactivas en personal de enfermería, de dos instituciones hospitalarias del área metropolitana de la ciudad de Medellín

Tabla 4. Variables asociadas con el consumo de sustancias psicoactivas en el personal de enfermería.

\begin{tabular}{|c|c|c|c|c|}
\hline Variable & $\begin{array}{c}\text { Consumo SPA } \\
n=106(\%)\end{array}$ & $\begin{array}{c}\text { No consumo de } \\
\text { SPA } \\
n=51(\%) \\
\end{array}$ & OR & IC \\
\hline \multicolumn{5}{|l|}{ Sexo } \\
\hline Femenino & $73(68.9)$ & $49(96.1)$ & 1 & \\
\hline Masculino & $33(31.1)$ & $2(3.9)$ & 11.07 & $2.54-48.28$ \\
\hline \multicolumn{5}{|l|}{ Grupo de edad } \\
\hline 20-35 años & $84(79.2)$ & $33(64.7)$ & 1 & \\
\hline$>36$ años & $22(20.8)$ & $18(35.3)$ & 0.48 & $0.22-1.00$ \\
\hline \multicolumn{5}{|l|}{ Servicio donde labora } \\
\hline Cirugía & $50(47.2)$ & $19(37.3)$ & 0.66 & $0.33-1.31$ \\
\hline Urgencias & $56(52.8)$ & $32(62.7)$ & 1 & \\
\hline \multicolumn{5}{|c|}{ Cargo que desempeña en la institución } \\
\hline Auxiliar de enfermería & $72(67.9)$ & $38(74.5)$ & 0.72 & $0.34-1-53$ \\
\hline Enfermero & $34(32.1)$ & $13(25.5)$ & 1 & \\
\hline \multicolumn{5}{|c|}{ Riesgo de depresión: escala de Zung } \\
\hline No & $96(90.6)$ & $46(90.2)$ & 0.95 & $0.31-2.96^{*}$ \\
\hline Sí & $10(9.4)$ & $5(9.8)$ & 1 & \\
\hline \multicolumn{5}{|l|}{ Autoreporte de estrés } \\
\hline No & $47(44.3)$ & $23(45)$ & 1.03 & $0.52-2.01$ \\
\hline Sí & $59(55.7)$ & $28(54.9)$ & 1 & \\
\hline \multicolumn{5}{|c|}{ Antecedente de trastorno mental } \\
\hline No & $94(88.7)$ & $47(92.2)$ & 1.50 & $0.45-4.90$ \\
\hline Sí & $12(11.3)$ & $4(7.8)$ & 1 & \\
\hline
\end{tabular}

*Estadístico de Fisher

Se encontró que el 9.5\% del personal de enfermería tiene riesgo de depresión, reporte similar a lo hallado por Mingote, quien estima que del 10\% al 12\% del personal de salud sanitario, perteneciente al sexo femenino, entre ellos enfermería, tienen, a lo largo de su carrera profesional, algún problema de salud mental, entre ellos depresión $\mathrm{y}$ ansiedad ${ }^{17}$. Esto puede ser posible por que las mujeres están expuestas a mayores niveles de estrés y ansiedad, por la carga emocional y las respuestas de afrontamiento ante situaciones estresantes o demandantes, que son más difíciles de sobrellevar ${ }^{18}$. Por otra parte, en relación con el consumo de sustancias psicoactivas el estudio identificó que ser hombre representa mayor riesgo para el consumo de sustancias psicoactivas, hallazgo similar al Estudio Nacional de Salud Mental del año 20154.

El estudio determinó que el $9.5 \%$ de las enfermeras encuestadas fue diagnosticado alguna vez en su vida con depresión. El estudio de Ohler halló prevalencias semejantes a este con el 9.4\%; los factores de riesgo reportados para sufrir depresión en ese personal fueron: consumo de cigarrillo, uso de antidepresivos, sobrecarga laboral y ser joven ${ }^{19}$. Al contrario del estudio realizado por Ohler,la investigación encontró que no existe relación entre el antecedente de trastorno mental con el consumo de sustancias psicoactivas. Es posible que la relación sea inversa y deben explorarse en otro estudio los factores asociados con la depresión en el personal de enfermería.

$\mathrm{Al}$ analizar el autoreporte de consumo de sustancias psicoactivas, se encontró que: la frecuencia de consumo de alcohol de las enfermeras (70.2\%) y auxiliares (62.7\%) es menor que la reportada en un estudio sobre consumo de sustancias en trabajadores de salud cuyo consumo en auxiliares de enfermería fue de (79.4\%) y para enfermeras de $(71.4 \%)^{20}$.

Respecto al tabaquismo, en México, Arenas et al., dio a conocer que en los hospitales de Guanajuato y Morelos, 
la prevalencia de consumo de tabaco es de $29.3 \%$ en enfermeras y $41.3 \%$ en médicos ${ }^{21}$. En cuanto al consumo de cigarrillo en la población estudiada, fue menor con el $20.4 \%$ y una dependencia leve con el $6.3 \%$ y la prevalencia difiere poco en cuanto al hallazgo del Instituto Mexicano del Seguro Social con el 17.6\% en personal de enfermería ${ }^{22}$.

En relación con el consumo de xantinas, la frecuencia más alta de consumo fue en la semana anterior con el 35.6 $\%$. En concordancia con la investigación "Sono, estresse e comportamientos compensatorios por enfermeras e parteiras australianas" el 93\% de las enfermeras consume cafeína considerada como la xantina de mayor consumo para lograr productividad labor ${ }^{23}$.

$\mathrm{Al}$ indagar por el consumo de sustancias ilícitas las de mayor consumo fueron: marihuana, benzodiacepinas sin indicación médica. Se sabe que la marihuana es la sustancia psicoactivas más consumida por la población general colombiana, por lo tanto es posible que sea la más consumida por el personal de salud ${ }^{4}$. En relación con el consumo de benzodiacepinas la literatura reporta que el $20 \%$ de las enfermeras recurre a medicamentos recetados como los sedantes para conciliar el sueño y disminuir niveles de estrés y ansiedad principalmente durante los turnos nocturnos y en horarios superiores a ocho horas de trabajo al día; de la población estudiada tres personas recurrieron al uso de sedantes con indicación médica y dos sin indicación médica ${ }^{24}$.

En la Universidad de Guayaquil se encontraron 17 estudiantes con consumo de benzodiacepinas sin prescripción, que se desempeñaban como auxiliares de enfermería, con turnos de 12 horas (70.6\%) en servicios de: emergencias, unidad de cuidados intensivos, áreas altamente críticas y de alto estrés como psiquiatría y en su mayoría en horarios nocturnos $(47.1 \%)^{24}$. En un estudio realizado en Bogotá el consumo de benzodiacepinas es del $10 \%$ en enfermeras y si se compara con este estudio, la prevalencia de consumo fue menor con el $0.6 \%{ }^{25}$.

Bell et al, reveló que el $10 \%$ de enfermeras usó potentes medicamentos anestésicos durante su carrera; los cuatro medicamentos más usados son: benzodiacepinas, óxido nitroso, opioides potentes y propofol. En la investigación "IPS de primer nivel de atención en consulta externa de Bogotá 2010-2011" el consumo de opioides en enfermeras es del $10 \%{ }^{25,26}$ es de resaltar que el presente estudio no halló consumo de opiáceos ni anestésicos.

No se indagó por dependencia de sustancias ilícitas; sin embargo, se puede inferir que no hubo dependencia ya que no se presentó consumo reciente (última semana, último día), diferente a los reportes de algunos estudios de Estados Unidos, en los que el 10\% de las enfermeras americanas es dependiente de las drogas, es decir, 300
000 enfermeras pueden ser adictas, por lo que existen diferentes programas orientados a cuidar a las cuidadoras con problemas de dependencia para favorecer su recuperación e integración a la vida social y laboral ${ }^{27,28}$.

Para la población colombiana trabajadora del área de la salud, las sustancias de mayor uso son: alcohol, cocaína, anfetaminas, morfina y anestésicos. En el presente estudio se encontró que el alcohol fue la sustancia más consumida: el 5\% tiene un consumo de riesgo, el 1.4\% consumo perjudicial, además hay bajos porcentajes de consumo de cocaína y anfetaminas y no se encontró consumo de morfina y anestésicos, a diferencia de los estudios anteriormente expuestos ${ }^{29}$.

Se considera que los resultados son significativos para estas dos instituciones porque permite conocer el riesgo de alcoholismo, tabaquismo, depresión y el consumo de sustancias psicoactivas. En Colombia, son limitados los estudios sobre el consumo de sustancias psicoactivas y enfermedad mental en personal de enfermería, por tanto, se recomienda realizar investigaciones en las instituciones hospitalarias y en los diferentes escenarios en los que participa el personal de enfermería, para hacer un diagnóstico de la problemática relacionada con el consumo en profesionales del área de la salud y tener más herramientas de análisis que permitan la construcción de programas de intervención en diferentes ámbitos.

Es importante diseñar y ejecutar investigaciones relacionadas con los factores de riesgo asociados con la depresión y consumo de sustancias psicoactivas en enfermería, estudiar el impacto de la depresión y ansiedad en la calidad de vida, determinar los factores protectores que potencian las habilidades sociales en el personal de enfermería y su capacidad de respuesta ante situaciones de estrés, así mismo, el impacto de programas de bienestar en la salud mental del trabajador.

Una de las limitantes del estudio fue no identificar el riesgo de adicción a drogas en el personal consumidor porque se calculó la prevalencia de consumo de sustancias ilícitas en vida, lo que dificultó la comparación con otros trabajos.

Se concluye que el estrés y la despersonalización son mayores en el personal de enfermería de este estudio que el reportado por otros investigadores. $\mathrm{El}$ consumo de sustancias y el riesgo de alcoholismo, tabaquismo y depresión es menor que en población general y similar a otros estudios específicos en personal de salud. Es importante que las instituciones propicien espacios de interacción familiar y afectiva que influyan en la salud mental de sus trabajadores, lo que puede incidir en la calidad de la atención que el personal de salud da a sus pacientes, así como la reducción de los niveles de estrés e insatisfacción. 


\section{DECLARACIÓN DE CONFLICTO DE INTERESES}

Los autores declaran no tener ningún conflicto de intereses.

\section{REFERENCIAS}

1. World Health Organization. Global Health Estimates 2013: Deaths by cause, age and sex; estimates for 2000-2012. Geneva: World Health Organization; 2014.

2. World Health Organization. Global Health Observatory: 7 Global status report on alcohol and health 2014. Geneva: World Health Organization. 2014.

3. World Health Organization. WHO global report on trends in tobacco smoking 2000-2025. Geneva: World Health Organization. 2015.

4. Ministerio de Salud y Protección Social, Colciencias, Pontificia Universidad Javeriana. Encuesta Nacional de Salud Mental 2015.Bogota: Ministerio de Salud y Protección Social; 2015.

5. Galvis Y, Posada J, Mejía R, Sierra GM, Montoya LP, Agudelo A. Primer Estudio Poblacional de Salud Mental Medellín, 2011-2012. Colombia: CES; 2012.

6. Baldisseri MR. Impaired healthcare professional. Crit Care Med. 2007; 35(2 Suppl):106-116.

7. Ministerio de la Protección Social, Dirección Nacional de Estupefacientes. Estudio Nacional de Consumo de Sustancias Psicoactivas en Colombia 2008: Informe final. Bogotá: Ministerio de la Protección Social; 2008.

8. Tiemi-Murofose N, Palucci MH. Trastornos mentales y de comportamiento en trabajadores de enfermería de 23 instituciones de salud en Brasil. Rev Enferm IMSS. 2005; 13(3):133-140.

9. Mininel VA, Baptista P, Felli VEA. Psychic workloads and strain processes in nursing workers of brazilian university hospitals. Rev Latino-Am Enfermagem 2011; 19(2):340-347.

10. Mejía N, Serna LE, Sierra T. Descripción de actitudes y prácticas en estilos de vida saludables en médicos y personal de enfermería. Metrosalud. Univ CES. 1997.

11. Díaz L, Campo A, Rueda G, Barros J. Propuesta de una versión abreviada de la escala de Zung para depresión. Colomb Med 2005;36(3):168-172.

12. Dalence T. Sistematización del taller sistemas de clasificación internacional y diagnósticos estadísticos. Quito, Ecuador: Proyecto Pradican; 2012.

13. Díaz CL, Ulloa CM, Taubert F, Amorim AL, Barcelos MC, Valenzuela SV, et al. El uso de drogas en el personal de enfermería. Cienc Enferm 2011; 17(2):37-45.

14. Chiang $\mathrm{Y}-\mathrm{M}, \mathrm{Chang} \mathrm{Y}$. Stress, depression, and intention to leave among nurses in different medical units: Implications for healthcare management/nursing practice. Health Policy 2012; 108(2-3):149-157.

15. Freitas $A R$, Carneseca EC, Paiva CE, Paiva BSR. Impact of a physical activity program on the anxiety, depression, occupational stress and burnout syndrome of nursing professionals. Rev Lat Am Enfermagem 2014; 22(2):332-336.

16. Tiemi-Murofose N, Palucci MH. Trastornos mentales y de comportamiento en trabajadores de enfermería de 23 instituciones de salud en Brasil. Rev Enferm IMSS. 2005; 13(3):133-140.

17. Mingote J. Las mujeres médicas son más propensas que los hombres a sufrir, estrés, depresión y ansiedad. Disponible en http://www.intramed.net/contenidover.asp?contenidoID $=45091$

18. Molina T, Gutiérrez A, Hernández L, Contreras C. Estrés psicosocial: algunos aspectos clínicos y experimentales. Anales de Psicología. 2008; 24(2):353-360.

19. Ohler MC, Kerr MS, Forbes DA. Depression in nurses. Can J Nurs Res. 2010; 42(3):66-82.

20. Rodríguez D. Prevalencia, nivel de dependencia y factores sociodemográfico según consumo de sustancias psicoactivas lícitas en trabajadores de la salud mental en la institución de tercer nivel de atención [Tesis de grado]. Bogotá: Universidad Pontificia Javeriana; 2008.

21. Arenas L, Jasso R, Martínez PC, Menjivar A. Prevalencia de tabaquismo de médicos y enfermeras en los estados de Morelos y Guanajuato. Rev Inst Nal Enf Resp Méx 2004; 17(4):261-265.

22. Fernández H, Escobedo J, Hernández D, Túdon J, Ramírez J, Benítez $M$, et al. Consumo de tabaco entre médicos y enfermeras. Salud Pública Méx 1997; 39(2):125-132.

23. Dorrian J, Paterson J, Dawson D, Pincombe J, Grech C, Rogers AE. Sleep, stress and compensatory behaviors in Australian nurses and midwives. Rev Saúde Pública. 2011; 45(5):922-930.

24. Paredes NP, Miasso AI, Tirapelli CR. Consumption of benzodiazepines without prescription among first-year nursing students at the University of Guayaquil, school of nursing, Ecuador. Rev Lat Am Enfermagem 2008; 16 (spe):634-639.

25. Lara C, Vargas GM, Salcedo A. Consumo de sustancias psicoactivas en profesionales de la salud (médicos y enfermeros) de dos IPS de primer nivel de atención en consulta externa de Bogotá. Rev Cienc Salud 2012;(Especial):87-100. 
26. Wright EL, McGuiness T, Moneyham LD, Schumacher JE, Zwerling A, Stullenbarger NEN. Opioid abuse among nurse anesthetists and anesthesiologists. AANAJ 2012; 80(2):120-128.

27. Trinkoff $A M$, Storr CL. Work schedule characteristics and substance use in nurses. Am J Ind Med 1998; 34(3):266-271.

28. Copp M. Drug addiction among nurses: Confronting a quiet epidemic. Modern Medicine. EEUU; 2009.

29. Ministerio de Protección Social. Estrategia de prevención en el ámbito laboral. Bogotá: Ministerio de Salud y Protección Social; 2007. 